\title{
Educación e INTERCULTURALIDAd EN OAXACA: AVANCES Y DESAFÍOS
}

\section{Education AND INTERCulturality in OAXaca: Progress and Challenges}

\section{Benjamín Maldonado Alvarado \\ benjaoax@yahoo.com.mx}

Currículo: doctor en Estudios Amerindios por la Universidad de Leiden, Holanda. Profesor de la Universidad Pedagógica Nacional Unidad 201. Sus líneas de investigación versan sobre educación comunitaria, etnografía de los pueblos indígenas, comunalidad y magonismo.

\section{Carlos Luis Maldonado Ramírez \\ lioslemsc@hotmail.com}

Currículo: maestro en Antropología Social en el área de Historia de la Educación, CIESAS Pacífico Sur. Miembro del cuerpo académico del Centro de Aprendizajes y Consultoría Educativa "Epistémica", Oaxaca. Profesor de la Universidad Mesoamericana, Oaxaca. Sus líneas de investigación versan sobre cultura escolar, investigación educativa, educación media superior y superior intercultural.

Recibido: 30 de agosto de 2017. Aceptado para su publicación: 23 de abril de 2018. Recuperado de: https://sinectica.iteso.mx/index.php/SINECTICA/article/view/788 DOI: 10.31391/S2007-7033(2018)0050-006

\section{Resumen}

Este artículo muestra la forma comunitaria que ha adoptado la interculturalidad en la educación oaxaqueña gracias a los logros del magisterio y los intelectuales indígenas. Los principales avances, garantizados en documentos oficiales, se concretan en el siglo XXI mediante la conformación de instituciones especializadas y en el diseño del modelo educativo comunitario, cuyas características principales son expuestas. El manuscrito revisa también los obstáculos actuales que enfrentan algunas de estas experiencias y presenta el análisis crítico de uno de los aprendizajes adquiridos en torno al manejo de los conocimientos comunitarios. Palabras clave: educación comunitaria, magisterio oaxaqueño, conocimiento local, interculturalidad.

\begin{abstract}
This article exposes the communitarian focus that interculturality has adopted in Oaxacan education thanks to the achievements of teachers and indigenous intellectuals. The main advances, guaranteed in official documents, have been consolidated in the 21st century through the creation of specialized institutions and the design of the community educational model, whose main characteristics are exposed here. We also reviews the current obstacles faced by some of these experiences and presents the critical analysis of one of the lessons learned about the management of community knowledge.
\end{abstract}

Keywords: communitarian education, Oaxacan teaching, local knowledge, interculturality. 


\section{INTRODUCCIÓN}

- n Oaxaca, la interculturalidad se ha concretado en la educación comunitaria. El modelo comunitario (en formación, distinto al del Consejo Nacional de Fomento Educativo) se perfila con base en una definición precisa de lo intercultural como un diálogo entre culturas. Este diálogo por construir no ocurre entre dos culturas, sino entre personas pertenecientes a culturas diferentes, y no puede haber diálogo entre desiguales, sobre todo si su relación es de dominación. El desafío de la interculturalidad está en lograr las condiciones para ese diálogo, que el Estado nacional mexicano rechaza e incluso combate por vocación.

Desde esa perspectiva, el alto grado de desarrollo político de organizaciones e intelectuales indígenas y no indígenas en décadas de movimiento social oaxaqueño ha permitido entender que la lucha no es por tener una educación intercultural genérica, sino por una educación comunitaria específica que forme a los niños y jóvenes que habrán de construir una sociedad intercultural; es decir, que el objetivo no es la educación intercultural, sino la sociedad mexicana y regional interculturales (Maldonado, 2016). La interculturalidad se percibe como un fin social, no como un medio, por lo que se pretende que la educación comunitaria fortalezca las raíces para luchar coherentemente por transformar la sociedad hacia su interculturalización. La educación comunitaria es intercultural porque promueve desde lo comunitario la interculturalidad.

Es importante señalar que en este artículo abordamos una propuesta educativa de la que hemos sido parte en su formulación teórica, diseño curricular y desarrollo docente desde hace más de veinte años, especialmente en experiencias de educación inicial, preescolar y primaria de la Coalición de Maestros y Promotores Indígenas de Oaxaca, en las secundarias comunitarias indígenas, en los bachilleratos integrales comunitarios, en el Colegio Superior para la Educación Integral Intercultural de Oaxaca y su Unidad de Estudios Superiores de Alotepec, y en la Escuela Normal Bilingüe e Intercultural de Oaxaca, y hemos realizado y publicado investigaciones y promovido estudios sobre varias de estas experiencias (ver referencias bibliográficas).

Vale la pena aclarar que no nos proponemos analizar aquí la relación y las diferencias entre educación comunitaria y educación intercultural en la práctica escolar, sino mostrar los avances que ha alcanzado el magisterio en documentos oficiales, al clarificar y fortalecer su propuesta político-pedagógica.

Así, el objetivo de la educación intercultural en pos de una sociedad intercultural es formar en tres dimensiones a personas que:

-Aprendan a ubicar y valorar la diversidad cultural.

- Puedan relacionarse con personas de otras culturas y tengan solidez en su formación cultural.

-Promuevan en la sociedad mexicana el diálogo entre culturas.

No se trata solo de un modelo educativo; es un proyecto formativo necesariamente político, cuya base de sustentación es el fortalecimiento de la cultura, lengua e identidad del estudiante para que pueda, sobre ese cimiento, acceder a conocimientos de otras culturas y que tengan sentido articulado con sus conocimientos cotidianos, y no los desplace. Con esto se pretende reforzar las identidades del estudiante y su conciencia como colonizado para que pueda ver, sentir y andar por el mundo "con los pies en la tierra", es decir, con la fuerza de su cultura originaria. 
Para lograrlo, las diversas experiencias de educación comunitaria comparten algunos elementos sustanciales, por ejemplo:

- La investigación como método de aprendizaje.

- Los conocimientos comunitarios como ejes de articulación con los conocimientos hegemónicos.

- La lengua originaria como vehículo de formación.

La existencia expansiva de escuelas y subsistemas de educación comunitaria en todos los niveles educativos permite y obliga a que en Oaxaca se trabaje en la formulación y ensayo de articulación que lleve a la integración de un sistema educativo comunitario (Maldonado, 2010), pero aún no se está haciendo.

\section{AVANCES: LAS ESCUELAS DE EDUCACIÓN COMUNITARIA}

La educación comunitaria en Oaxaca se ha concretado en numerosas experiencias en todos los niveles educativos a partir de este siglo, como mostramos en la siguiente tabla.

Tabla. Experiencias de educación comunitaria en Oaxaca y año de creación

\begin{tabular}{|c|c|c|}
\hline $\begin{array}{l}\text { Nivel } \\
\text { educativo }\end{array}$ & Educación pública & Educación privada \\
\hline Inicial & $\begin{array}{l}\text { Nidos de lengua (2008) y diplomado en } \\
\text { Educación Inicial Comunitaria }(2011-2012)^{A}\end{array}$ & No \\
\hline Preescolar & $\begin{array}{l}\text { Escuelas de la Coalición de Maestros y } \\
\text { Promotores Indígenas de Oaxaca } \\
\text { Escuelas de las jefaturas mixtecas de la } \\
\text { Dirección de Educación Indígena (DEI) } \\
\text { (Maestros de la Nación Ñu Savi) (2009) }\end{array}$ & No \\
\hline Primaria & $\begin{array}{c}\text { Escuelas de la Coalición de Maestros y Promo- } \\
\text { tores Indígenas de Oaxaca (CMPIO) } \\
\text { Escuelas de las jefaturas mixtecas de la DEI } \\
\text { (Maestros de la Nación Ñu Savi) (2009) }\end{array}$ & No \\
\hline Secundaria & Secundarias comunitarias indígenas $(2004)^{D}$ & No \\
\hline Bachillerato & $\begin{array}{c}\text { Bachillerato Integral Comunitario } \\
\text { Ayuujk Polivalente }(1996)^{\mathrm{E}} \\
\text { Bachilleratos integrales comunitarios }(2001)^{\mathrm{F}} \\
\text { Telebachilleratos comunitarios }(2013)^{\mathrm{G}}\end{array}$ & $\begin{array}{l}\text { Preparatoria “José Mart'” } \\
\text { de San Francisco } \\
\text { Ixhuatán }(1982)^{\mathrm{K}} \\
\text { Bachillerato de Asunción } \\
\text { Ixtaltepec }(1987)^{\mathrm{L}}\end{array}$ \\
\hline Universidad & $\begin{array}{c}\text { Escuela Normal Bilingüe } \\
\text { Intercultural de Oaxaca }(2000)^{\mathrm{H}} \\
\text { Unidad de Estudios Superiores } \\
\text { de Alotepec }(2011)^{\prime} \\
\text { Universidad Comunal Intercultural } \\
\text { del Cempoaltépetl (2012) } \\
\text { Maestría en Educación Comunal de la } \\
\text { Universidad Autónoma Benito Juárez de } \\
\text { Oaxaca (UABJO, 2016)* }\end{array}$ & $\begin{array}{l}\text { Instituto Superior } \\
\text { Intercultural Ayuuk } \\
\qquad(2006)^{\mathrm{M}}\end{array}$ \\
\hline
\end{tabular}


A. Meyer y Soberanes (2009), CMPIO (2009), Jiménez, Martínez, Mendoza y Meyer (2015)

B. Maldonado (2002), CMPIO (2003), Vargas (2005), Marshall (2008)

C. Maestros de la Nación Ñu Savi (2012)

D. IEEPO (2004), Wagner (2008), Motola (2009), Antolínez (2010), Maldonado (2011), Slutsky-Moore (2011), Ruiz y Quiroz (2014), Briseño (2015), Kreisel (2017)

E. BICAP (2001), González (2004), Comboni, Juárez y Tarrío (2005), Gallardo (2013), Morán (2013)

F. Amor (2004), Maldonado y Pérez (2004), CSEllO (2005), Pérez (2008), Pardo (2009), Ricco y Rebolledo (2010), Maldonado y García (2013)

G. DGB/SEMS (2016), Weiss (2017)

H. García (2003), García, Llaguno y Méndez (2004), Reyes y Vásquez (2008), Espinoza (2016)

I. Maldonado y García (2013), Maldonado, C. L. (2014), Faustino y Maldonado (2015), Maldonado, C. L. (2017)

J. Vargas, Vargas, Vásquez y Pérez (2008), Vargas (2014), Arguello (2016), Jablonska (2015)

K. Sanabria (2015), página web https://prepajosemarti.jimdo.com/

L. Rioja (2013)

M. CEA-UIIA (2006), Estrada (2008), Pastrana (2010), Rodríguez (2014), Lebrato (2016)

* http://www.ice.uabjo.mx/maestria-en-educacion-comunal

El número de estas escuelas es alto y va en crecimiento. Hay nidos de lengua en diez comunidades; la CMPIO coordina las actividades en más de 300 escuelas (preescolar y primaria) y centros de educación inicial; los Maestros de la Nación ÑuSavi trabajan en cientos de escuelas en las seis jefaturas de zonas de supervisión de educación indígena en la región mixteca; existen 11 secundarias comunitarias indígenas; hay 49 bachilleratos integrales comunitarios y 73 telebachilleratos comunitarios (Maldonado, 2017). En estas escuelas se forman estudiantes originarios de comunidades con la conducción de un magisterio organizado, el cual, además de su lucha laboral, realiza una enorme labor de movilización de conocimientos comunitarios a través del trabajo cotidiano en las aulas con base en el modelo de educación comunitaria (Maldonado, 2017).

\section{AVANCES: LO INTERCULTURAL EN LOS PLANES DE ESTUDIO Y EN LAS LEYES}

La precisión específica de lo comunitario como base de una educación intercultural está presente en los planes de estudio y en otros documentos oficiales. Algunos dejan ver con más claridad su definición:

1. La Universidad Comunal Intercultural del Cempoaltépetl, de la icónica comunidad mixe de Santa María Tlahuitoltepec, fue abierta en 2012 por acuerdo municipal con intelectuales mixes y ofrece tres carreras. En su definición señala que

es una Institución que retoma la filosofía de la Comunalidad, desde donde se define pedagógicamente para facilitar la formación del Humano-Pueblo (dualidad de identidad, de dignidad, de conciencia y de trascendencia comunitaria).

Forma profesionistas arraigados en sus pueblos, envueltos en su cultura y sus prácticas comunitarias, caracterizados en su personalidad, en sus actos y pensamientos, capaces de crear ideas, saberes y conocimientos con visión comunal. 
Desde esta perspectiva valora, respeta, fomenta y prepara para la convivencia intercultural (http://www.unicem.tlahuitoltepec.com/sobre.php).

2. Los bachilleratos integrales comunitarios operan desde 2001 con un modelo educativo indígena integral y fueron replicados por la Coordinación General de Educación Intercultural Bilingüe para sus bachilleratos interculturales. En el modelo vigente se señala:

La propuesta de identidad que se plantea desde el Bachillerato Integral Comunitario es una alternativa que da atención a las demandas sentidas de las comunidades y organizaciones indígenas, ya que además del conocimiento científico, reconoce e incorpora los saberes y valores comunitarios en los programas educativos en un marco de respeto entre las distintas culturas, lo cual representa el eje para fomentar una educación comunitaria desde una perspectiva intercultural; preservando la identidad cultural desde las lenguas indígenas, el modo de vida y organización de los pueblos indígenas, la preservación de las tradiciones y costumbres, y todo lo que encierra ese nuevo concepto cosmogónico que los intelectuales indígenas han llamado comunalidad (CSEIIO, 2005, pp. 9-10).

La promoción del diálogo intercultural es el imperativo propuesto para dirigir el proceso de enseñanza aprendizaje sobre todo en contextos multiculturales. La formación ciudadana exige el fomento de actitudes de respeto, aceptación y valoración de las distintas identidades que se manifiestan en una sociedad utilizando la diversidad como recurso. El Modelo Educativo Integral Indígena promueve la educación entre jóvenes de diferentes culturas y formas de ver la vida pero también fomenta el desarrollo de competencias interculturales para formar individuos flexibles de pensamiento que mantengan los valores de su cultura, además de reconocer lo que otras culturas ofrecen (CSEIIO, 2005, p. 31).

3. El Documento Base de Educación Indígena, formulado por maestros que estaban comisionados en la DEI del Instituto Estatal de Educación Pública de Oaxaca (IEEPO), rige desde 2012 los trabajos de cerca de cinco mil docentes. En él se define al diálogo intercultural como

el diálogo que establecen personas y grupos que pertenecen a culturas distintas para, entre otras cosas, explicarse mutuamente los puntos de vista diversos desde los que miran al mundo y sus cosas, sus prioridades, los conocimientos que valoran, los problemas que enfrentan o las relaciones que establecen entre unas cosas y otras. De esta manera, todos aprenden de todos y los horizontes de todos se ensanchan.

En el diálogo intercultural, los significados de unos arrojan nuevas luces sobre los significados de otros, sugieren otras maneras de organizar las cosas, abren caminos para establecer nuevas relaciones, invitan a la formulación de otras preguntas distintas y dibujan nuevas rutas de creación.

El diálogo intercultural se potencia cuando en las sociedades existe un marco legal que reconoce y valora la diversidad lingüística y cultural, que garantiza la igualdad de derechos y responsabilidades, que atiende a la equidad y a la inclusión y que propicia el acceso igualitario a las oportunidades.

El diálogo intercultural se potencia cuando se da en el seno de sociedades que tienen un fuerte y profundo contacto con sus propias culturas y conocen bien sus 
lenguas, que valoran y amplían sus vocabularios, que tienen presentes sus propias historias y legados ancestrales y los aprecian y que, al mismo tiempo, están abiertas a la creación, a la innovación y al aprendizaje (DEl, 2014, pp. 40-41).

4. El Plan para la Transformación de la Educación de Oaxaca (PTEO) es la alternativa que elaboró y acordó la Sección 22 del Sindicato Nacional de Trabajadores de la Educación con el gobierno ante la Alianza por la Calidad de la Educación, y es ahora su arma de lucha contra la reforma educativa en las trece mil escuelas adscritas al movimiento democrático de la Sección 22. Su visión de lo intercultural resalta su carácter crítico:

Los trabajadores de la educación del estado de Oaxaca plantean una propuesta educativa con enfoque comunitario intercultural crítico, que tiene como eje central la recuperación y articulación de los saberes comunitarios, para construir los currículos escolares que den sentido y significado al proceso formativo de los niños, jóvenes y adultos del estado; es decir, que se establezca un vínculo estrecho entre la vida de la comunidad, la vida escolar y del sujeto en formación, esto son parte esencial del proyecto educativo.

En la concepción de lo comunitario intercultural crítico que proponemos, se establece como principio la relación y el respeto entre culturas, la crítica y autocrítica como formas de empoderamiento de los sujetos en lo individual y colectivo, por lo que se plantea tener una base común entre ellas, la diversidad lingüística, el territorio, trabajo comunal, la fiesta y el poder ciudadano, además de la defensa de los derechos humanos, la preservación de los recursos naturales, la salud, la alimentación, educación, etc. (IEEPO/Sección 22, 2013, p. 26).

5. La Ley de Educación para el Estado Libre y Soberano de Oaxaca se aprobó en abril de 2016 con base en una iniciativa elaborada conjuntamente por la Sección 22 y el gobierno de Oaxaca. Pese a que incluye artículos para imponer la reforma educativa por encima de acuerdos estatales, tiene definiciones interesantes:

Interculturalidad: a la presencia e interacción equitativa de diversas culturas y la posibilidad de generar expresiones culturales compartidas, adquiridas por medio del diálogo y de una actitud de respeto mutuo, para erradicar la exclusión, la invisibilización, la discriminación, la intolerancia y la agresión como formas de trato a lo diverso (artículo 4ำ, fracción XVI).

Diversidad cultural: a la multiplicidad de formas en que se expresan y transmiten las culturas de los grupos y sociedades, las que se manifiestan de distintos modos de creación, producción, difusión, distribución y disfrute, cualesquiera que sean los medios y tecnologías utilizados (artículo 4ํ, fracción IX).

Lo más relevante desde el punto de vista del quehacer pedagógico es que establece a la educación comunitaria como el modelo adecuado para la realidad oaxaqueña:

Artículo 15. Además de lo dispuesto en el artículo anterior, también proporcionará educación comunitaria a los pueblos originarios de Oaxaca en el nivel de educa- 
ción básica y en otros niveles y modalidades cuya presencia en los contextos de los pueblos originarios sea significativa.

Toda la educación comunitaria del tipo básico y normal y demás para la formación de maestros, se impartirá atendiendo los planes y programas de estudios determinados por la Secretaría. El Gobierno del Estado propondrá los contenidos regionales que hayan de incluirse en dichos planes y programas.

Artículo 16. El Poder Ejecutivo reconoce que el modelo pedagógico de educación comunitaria es significativo y pertinente para la atención educativa de los pueblos originarios, para ello se procurara la creación de la modalidad de Secundaria Comunitaria de Educación Indígena dentro del sistema educativo estatal. La prestación de servicios en dicha modalidad, se realizará atendiendo los planes y programas determinados por la Secretaría.

Artículo 17. La educación comunitaria es un estadio de la educación bilingüe intercultural que reconoce los valores culturales de la Comunidad y la existencia de conocimientos propios como base para ofrecer educación pertinente a los pueblos originarios de la Entidad.

I. Los planes de estudio para la educación comunitaria distintos a los de educación básica y normal y demás para la formación de maestros de educación básica deben contemplar la existencia de una geografía ritual, de conocimientos ancestrales contenidos en las lenguas originarias y de conocimientos de su vida cotidiana; II. La educación comunitaria reconoce la existencia de portadores de conocimiento comunitario que forman parte del grupo social de pertenencia, $y$

III. La educación comunitaria reconoce la existencia de un sujeto comunitario que se contrapone con el sujeto individual.

Su misma confusión de lo intercultural con lo comunitario es interesante y abre perspectivas de trabajo:

Artículo 19. Es obligación del Gobierno del Estado impartir educación intercultural para todos, en el caso de los pueblos originarios además será bilingüe, diseñando planes y programas de estudio distintos a la educación básica y normal y demás para la formación de maestros de educación básica, que integren conocimientos, tecnologías y sistemas de valores correspondientes a las culturas de la entidad, respetándose los derechos lingüísticos de los niños hablantes de lengua originaria. Esta enseñanza deberá impartirse en la lengua originaria y en español.

Toda la educación intercultural del tipo básico y normal y demás para la formación de maestros, se impartirá atendiendo los planes y programas de estudios determinados por la autoridad educativa federal. El Gobierno del Estado propondrá a la Secretaría los contenidos regionales que hayan de incluirse en dichos planes y programas.

Deberá considerarse que los docentes que trabajen en los pueblos y comunidades indígenas del Estado de Oaxaca hablen dicha lengua y que los medios de comunicación locales fortalezcan todas las lenguas y en particular las que se encuentran en vías de extinción.

Artículo 64. Los proyectos educativos y las aportaciones de los especialistas ayudarán a recuperar los conocimientos regionales, para su sistematización e integración en los programas de estudio, que permitirán la atención de la diversidad y, por ende, favorecerán la interculturalidad. 
6. Esto no es ajeno ni siquiera a la misma reforma educativa, que en su "Ruta para la implementación del modelo educativo", se ve obligada a reconocer (aunque sea la única mención en sus 160 páginas), que

una condición necesaria para la correcta implementación del Modelo Educativo y el Currículo actualizado de la EMS, es proveer ambientes pertinentes para el aprendizaje y entornos favorables para el desarrollo integral de los jóvenes, que incidan positivamente en el compromiso de los estudiantes con su aprendizaje, y articulen los diferentes elementos del currículo, incluidos aspectos como los siguientes: ... El fomento del diálogo intercultural y el desarrollo de una cultura de inclusión (SEP, 2017, p. 56).

Es evidente que los avances de la educación comunitaria han creado las condiciones reales propicias para su expansión y florecimiento, y se cuenta con aspectos realmente favorables de contexto. Para aprovecharlo, habrá que resolver los desafíos.

\section{DESAFÍOS: CRISIS INTERNAS Y EL CONTEXTO DE LA REFORMA}

Podemos ubicar dos ámbitos de los desafíos a los que se enfrenta la educación comunitaria oaxaqueña actualmente: los institucionales y los teóricos. Entre los primeros destacan la crisis de instituciones de educación básica, agudizada por la reforma educativa, y en educación media superior y superior, la pérdida de rumbo del Colegio Superior para la Educación Integral Intercultural de Oaxaca (CSEIIO). Entre los segundos se encuentran las carencias en el manejo de conocimientos comunitarios.

La reforma educativa del presidente Enrique Peña Nieto llegó a Oaxaca en momentos de crisis en varias de las instituciones de educación comunitaria:

1. Desde 2011, la CMPIO vive una crisis derivada de diferencias entre sus fundadores en 1974, hoy jubilados, y la directiva actual, sobre todo en cuanto al manejo de los bienes institucionales. Esta división ha afectado la continuidad de proyectos de trabajo como los nidos de lengua, que no han crecido o, incluso, decaen, así como la continuidad en la formación en educación inicial comunitaria, que no ha logrado desarrollar todo su potencial.

La CMPIO, que agrupa a unos mil quinientos profesores en más de trescientas escuelas, ha sido fundamental en el desarrollo del movimiento sindical y pedagógico de la Sección 22 y ha jugado un papel relevante en la formación e impulso del Congreso Nacional de Educación Indígena e Intercultural (CNEII) y del Foro de la Niñez Oaxaqueña.

2. Las Secundarias Comunitarias Indígenas tienen el modelo más innovador y propositivo en la educación comunitaria oaxaqueña, el cual ha servido de base para la formulación del PTEO y para los trabajos de los Maestros de la Nación ÑuSavi. Inició sus actividades en 2004 como resultado de una propuesta elaborada y gestionada por la CMPIO con el IEEPO, y a la fecha cuenta apenas con once escuelas por la falta de apoyo del IEEPO, que no lo ha aceptado como subsistema estatal y no hay voluntad para resolver enredos administrativos, principalmente de tipos de plaza.

Luego de un periodo de ascenso, en el cual el proyecto se desarrollaba en las escuelas y los docentes se formaban durante las va- 
caciones, se perdió ritmo a raíz de la muerte de uno de sus creadores, Luis Madrigal Simancas. Se relajó el ritmo de la formación y disminuyó la presencia propositiva de la coordinación del proyecto en las escuelas; con ello, la innovación ha ido cediendo espacio.

3. Respecto a los Maestros de la Nación ÑuSavi, atraídos por las características y resultados del modelo de las secundarias comunitarias, los cientos de maestros que integran las seis zonas de supervisión de educación indígena en la región mixteca se organizaron para aprender del modelo y reorganizar el trabajo en sus aulas; esto, a fin de generar un currículo apropiado para trabajar en territorio mixteco. También recurrieron a los aprendizajes obtenidos en los trabajos realizados desde 1999 dentro de la Marcha de las Identidades Étnicas y continuados con los congresos étnicos, promovidos desde la DEI del IEEPO. Desde finales de 2009 se organizaron regionalmente, pusieron en práctica sus aprendizajes del modelo comunitario y formularon una propuesta curricular en 2012, con la que trabajan.

\section{La DEI ha generado propuestas importantes de trabajo en las aulas:}

La continuidad del Proyecto para el Desarrollo de una Educación Bilingüe e Intercultural del Estado de Oaxaca se da con el proyecto denominado: "Marcha de las Identidades Étnicas por una Educación Bilingüe Intercultural" que inicia en enero de 1999, en la comunidad de Patio Iglesias, Huautla de Jiménez, abarcando siete Jefaturas de Zonas de Supervisión, hasta septiembre del 2000. En el año 2001 se continúa desarrollando acciones del proyecto cubriendo las 16 Jefaturas restantes.

Primera fase: ésta consistió en la captación y sistematización de los saberes comunitarios a través de la indagación y registro de la observación de los temas generadores con sus siete mundos que son: natural, social, matemático, lingüístico, arte, corporal e imaginario.

Segunda fase: Se llevó a cabo la sistematización de los saberes comunitarios, se clasificaron en conocimientos, valores, habilidades, destrezas y actitudes susceptibles de llevarlos al aula como contenidos educativos propios de cada pueblo originario. Tercera fase: se realizaron ocho congresos de los tres niveles educativos en las siguientes etapas: zonal, jefatura y estatal, abordando ocho temas: 1 ) trazar y consensuar los ejes rectores. 2) definir y caracterizar la educación bilingüe e intercultural. 3) fundamentación de una política del lenguaje. 4) sintetizar y definir los valores, actitudes, destrezas y habilidades. 5) aprobar las bases para una nueva relación de los pueblos originarios con la sociedad nacional. 6) reconocer la comunalidad como raíz y razón de los pueblos. 7) definir la postura frente a la política indigenista. 8) conservar las posturas de las culturas originarias frente a la globalización.

En febrero de 2003 se realizó el primer Congreso Étnico, cuyo propósito central fue captar y sistematizar saberes comunitarios con base en los trabajos de la primera y segunda fase, desde la experiencia que se genera en nuestros pueblos originarios. En este evento participaron activamente personas caracterizadas de cada nivel educativo de 14 pueblos originarios del estado de Oaxaca: Huaves, Chontales, Zapotecas, Mixtecos, Mazatecos, Chinantecos, Triquis, Chatinos, Nahuas, Chocholtecos, Cuicatecos, Amuzgos, Mixes y Zoques, al igual maestros, padres de familia, Autoridades gubernamentales, especialistas y compañeros de la Sección 
XXII, abarcando las 23 Jefaturas de Zonas de Supervisión, 196 mesas Técnicas de Jefatura y Supervisiones Escolares, 12,000 elementos entre directivos y docentes de los niveles de Educación Inicial, Preescolar, Primaria y Servicios de Extensión Educativa, encaminados a indagar y sistematizar los saberes comunitarios en el contexto de la diversidad cultural lingüística con fundamento teórico y jurídico.

La indagación de los saberes comunitarios condujo a clasificarlos por mundos: natural, social, matemático, lingüístico, arte, corporal e imaginario. A partir de las observaciones obtenidas en la investigación, se elaboraron campos semánticos para sistematizar los conocimientos culturales, mediante tres categorías: saberes cotidianos, contenidos étnicos y científicos.

El Segundo Congreso Étnico Estatal se realizó en mayo de 2007, bajo los siguientes ejes temáticos: a) Construcción colectiva de contenidos de los saberes comunitarios, de los pueblos originarios. B) Organización de contenidos por niveles educativos y pueblos originarios, sistematizándolos por mundos, campos semánticos y ejes temáticos en las asignaturas. C) Correlación y articulación de los saberes comunitarios, con los planes y programas Nacionales. D) Diseño de metodologías y estrategias de trabajo docente, así también la producción de materiales educativos bilingües e interculturales. E) Impulsar la construcción del diseño curricular que incida en los Niveles Educativos de Educación Inicial, Preescolar y Primaria, como un primer acercamiento al Currículum Escolar de una Educación Bilingüe e Intercultural (Maestros de la Nación ÑuSavi, 2012, pp. 20-22).

Luego, la DEI elaboró el documento base en 2012 y dejó inconclusa una propuesta curricular de educación comunitaria indígena al momento en que la reforma educativa penetró en Oaxaca.

La reforma educativa del presidente Enrique Peña Nieto fue aprobada en febrero de 2013 y en noviembre se creó la Coordinación General de Servicio Profesional Docente de la SEP, mientras que el Instituto Nacional para la Evaluación de la Educación, creado en 2002, fue reformado como autónomo en febrero de 2013. Con esto, la reforma tenía sus dos puntas de lanza institucionales listas, y se inició un contradictorio proceso de consulta a comunidades indígenas, y la cuestionada evaluación. En esos años, la Sección 22 y el gobierno estatal probaron el PTEO y elaboraron una iniciativa de ley de educación. En 2015 irrumpió la reforma en Oaxaca, principalmente con la recreación del IEEPO en julio y la ruptura de acuerdos con la Sección 22.

El golpeo constante al magisterio desalentó los procesos de educación comunitaria al obligar a los maestros a tener una actitud defensiva, pero movilizada y dedicarse a librar los ataques de la reforma. Tanto para el IEEPO como para el magisterio, la educación pasó a segundo plano a partir de 2015, lo prioritario era imponer la reforma y defenderse de ella. Sin embargo, el PTEO es ahora el arma de lucha del magisterio contra la reforma, una propuesta basada en la educación comunitaria ya probada en miles de aulas.

El caso de la DEI es paradigmático: su fuerte dinámica de trabajo se interrumpió en julio de 2015 cuando el IEEPO regresó a sus centros de trabajo a todos los maestros comisionados en la oficina central, y los sustituyó por administradores sin experiencia. Con ello se perdió el impulso institucional a la educación comunitaria, pero se mantuvo vivo en la conformación de una DEI en resistencia, que cuenta con el apoyo de la Sección 22 hasta la fecha. Todo esto hizo que la educación comunitaria adquiriera un rostro claramente contrahegemónico. 


\section{DESAFí́OS: EL NAUFRAGIO INSTITUCIONAL, EL CSEIIO DURANTE EL RÉGIMEN DE LA ESPERANZA} El CSEIIO es una institución pública descentralizada creada en 2003 para tener a su cargo planteles de educación comunitaria de nivel medio superior y superior. En sus primeros siete años se dedicó a dar forma al proyecto y sobrevivió al 2006, pues de los cinco directores generales que tuvo en ese lapso, la tercera provocó un movimiento de oposición que la destituyó y el quinto tuvo la posibilidad de darle forma y solidez al CSEIIO durante los tres años (2007-2010) de su administración (Maldonado y García, 2013). Al tomar posesión en diciembre de 2010, Gabino Cué (primer gobernador de oposición en la historia de Oaxaca) nombró a un nuevo director, quien, junto con los otros dos directores que nombraría Cué después, se dedicó a saquear los recursos del Colegio y a desfigurar el proyecto académico que le daba sentido e identidad.

EI CSEIIO administra 49 bachilleratos integrales comunitarios a los que asisten más de cinco mil jóvenes indígenas y una unidad de estudios superiores que tiene una sola licenciatura. Durante su administración (2014-2017), el profesor Guillermo Bernal encargó la transformación radical de los planes de estudio universitario y de bachillerato a una misma "especialista". El resultado fue, en el caso de bachillerato, la aprobación apresurada de los dos primeros semestres del nuevo plan para iniciar el ciclo 2014-2015, cuando luego de años de trabajar en ello no estaba listo aún ni el plan ni los programas de trabajo de los seis semestres (módulos). El nuevo plan se "adecuaba" a un modelo por competencias y encajaba en los dos últimos módulos varias opciones de formación para el trabajo sin considerar créditos suficientes $y$, sobre todo, sin haber maestros adecuados, por lo que se basaba en improvisarlos (Maldonado, 2017).

En el caso de estudios superiores, el profesor Bernal y su equipo decidieron la transformación drástica del plan de estudios de la licenciatura en Educación Media Superior Comunitaria, de 71 materias, por el de la licenciatura en Educación Intercultural Comunitaria, de $\mathbf{4 8}$ materias. Lo grave del cambio fue que lo impusieron cuando aún no egresaba la primera generación, es decir, sin una evaluación mínima se decidió la supresión de un plan y su sustitución. Esto ocurrió en 2015 cuando los 80 estudiantes de la primera generación estaban en el último semestre, en otras palabras, cuando habían cursado 70 materias y tenían calificaciones registradas de ellas; por ello, se les avisó que se titularían con un plan de solo 48 materias (Maldonado, C., 2017).

Los cambios en el horizonte de sentido del CSEIIO, expresado en sus nuevos planes de estudio, sumados al autoritarismo y desconocimiento de la educación comunitaria de los últimos directores generales y su equipo, avalados por su consejo directivo, han sumido al CSEIIO en una profunda contradicción: los esfuerzos por lograr una educación de calidad no corresponden al sentido original de la institución, de modo que esa institución especializada en educación comunitaria se desentiende cada vez más de su misión y se aleja de un servicio adecuado para los pueblos indígenas.

\section{DESAFÍOS: CONOCIMIENTOS COMUNITARIOS Y HORIZONTE EPISTEMOLÓGICO}

Uno de los principales aprendizajes alcanzados en Oaxaca es empezar a ver que la forma adecuada de la educación intercultural o comunitaria consiste en la separación de conocimientos, de manera similar a la práctica de educación bilingüe 
mediante separación de lenguas. En Oaxaca esto lo trabajó y difundió el preescolar ikoots de San Mateo del Mar, perteneciente a la CMPIO (Aguilar, s.f.), y su éxito nos ha permitido pensar una analogía con los conocimientos: ensayar la educación comunitaria o intercultural mediante separación de conocimientos. Sin embargo, separar conocimientos implica de modo necesario trabajar los conocimientos comunitarios en su matriz cultural y no en la hegemónica, como se ha hecho en forma regular. Para profundizar en ello, recurrimos a una experiencia peruana.

Las propuestas educativas del Proyecto Andino de Tecnología Campesina (Pratec) ilustran el modo de abordar en el aula y fuera de ella los dos tipos de conocimiento, cuyo primer paso debe ser la formación sólida de los estudiantes en su propia cultura para, posteriormente, aprender elementos de otras culturas y desarrollar un contraste entre ambas formas de aproximarse a la realidad. Desde la perspectiva de Grimaldo Rengifo, el contraste entre conocimientos permite generar equivalencias entre ellos:

... para que se enfatice la equivalencia de saberes, cada tema debe ser presentado de manera contrastada [...] se supera así el denominado "plano inclinado cognoscitivo" que acostumbra colocar los saberes locales en el peldaño inicial, pero se debe tener cuidado de no reproducir el espíritu dualista que anima a la escuela moderna respecto del saber local, esta vez al revés (Rengifo, 2005, p. 16).

Con un ejemplo concreto, expone la forma de llevar a cabo dicho contraste epistemológico por medio de uno de los elementos principales que caracteriza e identifica a las culturas andinas y mesoamericanas: el vínculo entre el hombre y la naturaleza, expresado a través del trabajo y la relación con la tierra:

Si la chacra es vivenciada andinamente como persona, debe mostrarse a los alumnos esta concepción contrastándola con la perspectiva científica, dentro de la cual la chacra es un recurso a disposición del hombre. Esto permite dos cosas: mostrar el saber en su contexto cultural y hacerlo equivalente a cualquier tradición cognoscitiva existente (Rengifo, 2005, p. 16).

En Perú se ha logrado que, dentro del currículo oficial, se cuente con diez horas para el desarrollo del currículo local (Pardo y Roddo, 2008). Si bien la ampliación del tiempo es una de las demandas, la forma de aprovecharlo implica un trabajo integral entre autoridades locales, padres de familia, mediadores culturales (docentes) y alumnos, cuya resignificación del aparato escolar hace pensar más allá de la escuela, al encontrar en la comunidad una escuela más grande, donde se presenta el espacio natural de los conocimientos locales.

En el plano vivencial se encuentran los ritmos, tiempos, espacios y características del conocimiento local; aprender-haciendo en la comunidad evita la "teatralización de una actividad cultural referida a la dinámica comunal" (Pardo y Roddo, 2008, p. 112). En un estudio elaborado por Mendoza et al. (2008) se ilustra la experiencia del Centro de Estudios Andinos "Vida Dulce", la cual da cuenta de la incorporación de "saberes de crianza de los Chacra Huertos Campesinos al currículo por una doble vía: de la comunidad a la escuela, y de la escuela a la comunidad" (p. 89).

Además, hemos visto que la fuerza y profundidad del conocimiento comunitario está en la lengua originaria como vehículo de construcción de conocimientos. 
Por ejemplo, cuando se pide a un estudiante que diga cómo se llaman los momentos del día en su lengua, se le orilla a un ejercicio forzado de traducción, pero si se le pide que diga cómo llaman en su lengua a los momentos del día, entonces sin duda hará una lista más larga que madrugada, amanecer, mediodía, etcétera, y al tener nombres distintos, tendrán también significados diferentes a los hegemónicos. La lengua es el vehículo que remite a la matriz original de los conocimientos, por lo que la educación intercultural debe avanzar hacia la profundización del conocimiento comunitario en una matriz propia, no hegemónica, y hacerlo en su lengua, con sus sabios.

\section{Conclusión}

Para llevar a cabo estas iniciativas, al igual que en el caso de la educación comunitaria oaxaqueña, debe existir un vínculo y una participación directa de autoridades locales, docentes, gente de la comunidad y estudiantes. En el contexto oaxaqueño, la escuela es resignificada y abierta hacia la comunidad. Se procura la descolonización del docente y su práctica como figura de poder, y su función cambia a mediador, acompañante o facilitador. Se visibiliza el conocimiento de las personas de la comunidad y pasan a desempeñar una labor fundamental en el aprendizaje de los estudiantes. El aula es la comunidad, no como intento de encasillar a la comunidad, sino como espacio propicio para el aprendizaje. Los procesos pedagógicos deben partir de la vida comunal y son experienciales, pues implican de modo necesario un aprender-haciendo. Los conocimientos locales expresan realidades culturalmente situadas que sirven para comprender el mundo; por tanto, no son excluyentes de los conocimientos universales u occidentales; ambos implican, en la búsqueda de una equivalencia de conocimientos, formas diversas de ser y estar en el mundo.

Si la diversificación curricular ha sido impulsada como una respuesta a la homogeneidad, y en esto puede caber incluso la llamada autonomía curricular de la reforma, deberíamos pensarla como una posibilidad de diálogo entre culturas, de acuerdo con Rengifo (2005). Es mucho lo que se ha avanzado y aprendido con la educación comunitaria oaxaqueña, y también son increíbles los obstáculos a vencer. Hace falta analizar los aprendizajes a fondo y reorientar lo necesario para seguir por caminos adecuados.

Los esfuerzos por preparar niños y jóvenes, y también padres de familia, para que tengan bases sólidas a fin de luchar por construir un México intercultural han florecido en condiciones adversas. Se esperaba que eso cambiara luego de dos gobernadores priistas. Durante el régimen de José Murat (1998-2004) lograron nacer la mayoría de las experiencias de educación comunitaria; continuaron durante la administración de Ulises Ruiz (2004-2010); llegaron al régimen de la esperanza suponiendo que tendrían condiciones para desarrollar su potencial, pero el gobierno de Gabino Cué (2010-2016) traicionó la esperanza. Sin embargo, la educación comunitaria sigue con vida y posibilidades de fortalecimiento gracias al magisterio democrático, y no es imposible que el régimen de Alejandro Murat (2016-2022) logre establecer acuerdos constructivos con la Sección 22, si el nuevo gobierno federal no sigue obligando al gobierno oaxaqueño a lo contrario. Mientras, los maestros oaxaqueños continúan trabajando en las aulas con base en los lineamientos del PTEO y la educación comunitaria, y resistiendo los embates laborales de la reforma educativa, aunque postergando la profundización en el campo epistemológico. 


\section{REFERENCIAS BIBLIOGRÁFICAS}

Aguilar, M. (s.f.). Educación y desarrollo lingüístico en la diversidad cultural. Xictli. Recuperado de http://189.208.102.74/u094/revista/56/06.html

Amor, I. (2004). Modelo educativo de nivel medio superior: el Bachillerato Integral Comunitario de Oaxaca. Tesis de maestría en Ingeniería de Sistemas, IPN. Recuperado de http://tesis.ipn.mx/bitstream/handle/123456789/15746/Modelo\%20 educativo\%20de\%20nivel\%20medio\%20superior\%20-\%20bachillerato\%20integral\%20comunitario\%20de\%20oaxaca.pdf?sequence=1

Antolínez, I. (2010, febrero). Una mirada a la secundaria comunitaria de San Andrés Solaga, análisis FODA. Educación Comunal, núm. 3, pp. 47-81.

Arguello, A. (2016). Pedagogía mixe: contribuciones para una filosofía (decolonial) de la educación desde las Américas. Estudios Pedagógicos, vol. XLII, núm. 3, pp. 429-447. Recuperado de http://mingaonline.uach.cl/pdf/estped/ v42n3/art23.pdf

BICAP (2001). La voz y la palabra del pueblo ayuujk. México: UPN/BICAP/Miguel Ángel Porrúa Ed.

Briseño, J. (2015). Entrevistas comunitarias, la recuperación del conocimiento comunitario desde la escuela. El caso de las Secundarias Comunitarias Indígenas, Oaxaca, México. Archivos Analíticos de Políticas Educativas, vol. 23, núm. 95. http:// dx.doi.org/10.14507/epaa.v23.2081

CEA-UIIA (2006). Hacia dónde vamos. Un diagnóstico de la región mixe. México: CDI/ Sistema Universitario Jesuita.

CMPIO (2009). Nidos de lengua. Educación Comunal, núm. 2, pp. 39-52.

CMPIO (2003). Tequio pedagógico. Colaboración pedagógica en comunidad. Oaxaca: Coalición de Maestros y Promotores Indígenas de Oaxaca.

Comboni, S., Juárez, J. M. y Tarrío, M. (2005). Resurgimiento cultural indígena: el pueblo Ayuujk de Santa María Tlahuitoltepec, Mixe, Oaxaca. El Bachillerato Integral Comunitario Ayuujk Polivalente. Revista Latinoamericana de Estudios Educativos, vol. XXXV, núm. 1-2, pp. 181-208. Recuperado de http://www.redalyc.org/ pdf/270/27035207.pdf

CSEIIO (2005). Modelo de educación integral indígena. Oaxaca: Colegio Superior para la Educación Integral Intercultural de Oaxaca.

DEI (2014). Documento base de la educación de los pueblos originarios. Oaxaca: Dirección de Educación Indígena del IEEPO.

DGB/SEMS (2016). Telebachillerato comunitario. Documento base. México: Dirección General de Bachillerato de la SEP.

Espinoza, M. (2016, noviembre). La experiencia de la Escuela Normal Bilingüe e Intercultural de Oaxaca sobre el Medio Ambiente como elemento de recuperación de contenidos académicos. Ponencia presentada en el Primer Congreso Nacional de Educación Ambiental para la Sustentabilidad, Tuxtla Gutiérrez, Chiapas. Recuperado de http://www.anea.org.mx/CongresoEAS/Docs/323PINTR-EspinosaRivas.pdf

Estrada, G. (2008). La experiencia del Centro de Estudios Ayuuk-Universidad Indígena Intercultural Ayuuk. En D. Mato. Diversidad cultural e interculturalidad en educación superior (pp. 371-380). Caracas: Instituto Internacional para la Educación Superior en América Latina y el Caribe.

Faustino, C. y Maldonado B. (comps.) (2015). Investigaciones y debates en la UESA. Tomo I: Los académicos. Oaxaca: CSEIIO (edición virtual). 
Gallardo, C. (2013). Educación escolar, política comunal e ideología étnica: los maestros indígenas de Santa María Tlahuitoltepec. Tesis de maestría en Antropología Social, CIESAS Pacífico Sur, Oaxaca.

García, E., Llaguno, I. y Méndez, C. (2004). La Escuela Normal Bilingüe e Intercultural de Oaxaca. En L. Meyer y B. Maldonado (comps.). Entre la normatividad y la comunalidad. Experiencias educativas innovadoras del Oaxaca indígena actual (pp. 475-510). Oaxaca: Fondo Editorial del Instituto Estatal de Educación Pública de Oaxaca.

García, V. (comp.). (2003). Escuela Normal Bilingüe Intercultural de Oaxaca. Guía académica 2002-2003. Oaxaca: Fondo editorial del IEEPO.

González, E. (2004). Significados escolares en un bachillerato mixe. México: Coordinación General de Educación Intercultural Bilingüe de la SEP.

IEEPO (2004). Proyecto de secundaria para la atención de comunidades pertenecientes a los pueblos originarios del estado de Oaxaca. Oaxaca: Instituto Estatal de Educación Pública de Oaxaca.

IEEPO/Sección 22 (2013). Plan para la Transformación de la Educación de Oaxaca. Recuperado de http://www.transformacion-educativa.com/attachments/article/94/ CuadernilloPTEO.pdf

Jablonska, A. (2015). La Universidad Comunal Intercultural del Cempoaltépetl: un proyecto político, cultural y pedagógico. En P. Medina (coord.). Pedagogías insumisas: movimientos político-pedagógicos y memorias colectivas de educaciones otras en América Latina (pp. 235-254). México: Unicach/Cesmeca/Juan Pablos Editor.

Jiménez, J., Martínez, L., Mendoza, J. y Meyer, L. (2015). Análisis de las actividades de niños de educación inicial en pueblos originarios de Oaxaca. Anthropologica, vol. 33, núm. 35, pp. 141-172. Recuperado de http://www.scielo.org.pe/scielo. php?pid=S0254-92122015000200007\&script=sci_arttext

Kreisel, M. (2017). Las secundarias comunitarias indígenas de Oaxaca: resignificaciones de la educación escolarizada desde una propuesta alternativa para la formación de los jóvenes. Tesis de doctorado en Investigación Educativa, Universidad Veracruzana. Recuperado de https://www.uv.mx/pdie/files/2015/06/Tesis_Maike-Kreisel.pdf

Lebrato, M. (2016). Diversidad epistemológica y praxis indígena en la educación superior intercultural en México: un caso de estudio en el Instituto Superior Intercultural Ayuuk. Revista Mexicana de Investigación Educativa, vol. 21, núm. 70, pp. 785-807. Recuperado de http://www.scielo.org.mx/scielo.php?script=sci_arttext \&pid=S1405-66662016000300785

Ley de Educación para el Estado Libre y Soberano de Oaxaca (2016). Recuperado de https://legislacion.vlex.com.mx/vid/ley-educacion-libre-soberano-637484173

Maestros de la Nación Ñu Savi (2012). Currícula comunal para la educación de los pueblos originarios de Oaxaca. Oaxaca: Consejo Técnico de la Nación Ñu Savi.

Maldonado, B. (2017). Movimiento social y movilización de conocimientos comunitarios en Oaxaca. México: Universidad Iberoamericana (en prensa).

Maldonado, B. (2016). Hacia un país plural: educación comunitaria en Oaxaca frente a la política de interculturalidad cero. Liminar, vol. XIV, núm. 1, pp. 47-59.

Maldonado, B. (coord.) (2011). La investigación como proyecto de aprendizaje en la Secundaria Comunitaria Indígena de San Andrés Solaga, Oaxaca. Informe para la Red Latinoamericana de Convivencia Escolar. Oaxaca: Coordinación Estatal de Escuelas de Educación Secundaria Comunitaria Indígena. 
Maldonado, B. (2010). Comunidad, comunalidad y colonialismo. La nueva educación comunitaria y su contexto. Tesis de doctorado en Estudios Amerindios, Universidad de Leiden, Holanda.

Maldonado, B. (2002). Los indios en las aulas. Dinámica de dominación y resistencia en Oaxaca. México: INAH.

Maldonado, B. y Pérez, F. (coords.) (2004). Educación integral para los pueblos indígenas de Oaxaca. Los primeros pasos de un proyecto innovador: el Bachillerato Integral Comunitario 2001-2004. Tomo III de la colección La marcha educativa en Oaxaca 1998-2004. Oaxaca: Instituto Estatal de Educación Pública de Oaxaca.

Maldonado, C. L. (2017), La configuración de la cultura escolar en el proyecto educativo de la UESA-LEMSC. Procesos de apropiación y resignificación de lo comunitario en contextos de conflicto. Tesis de maestría en Antropología Social, CIESAS, Oaxaca.

Maldonado, C. L. (2014), Política intercultural, educación comunitaria y lengua originaria. Historias de vida escolar de los jóvenes de la UESA-LEMSC. Tesis de licenciatura en Ciencias de la Educación. Oaxaca: Instituto de Ciencias de la Educación de la UABJO.

Maldonado, C. L. y García, E. (coords.) (2013). 10 años del CSEllO desde una mirada universitaria. México: UESA. Recuperado de http://cseiio.edu.mx/attciudadana/ librodiezanos_cseiio.pdf

Marshall, A. (2008). Fighting the system from within: CMPIO and education reform. Educación Comunal, núm. 1, pp. 87-112.

Mendoza, A. et al. (2008). La Chacra Huerta Campesina y su incorporación al currículo educativo. En Diálogo de saberes y escuela rural andina (pp. 81-106). Lima: Proyecto Andino de Tecnologías Campesinas.

Meyer, L. y Soberanes, F. (2009). El nido de lengua. Orientación para sus guías. Oaxaca: CMPIO/CNEII/CSEIIO.

Morán, N. (2013). El Modelo Intercultural del Bachillerato Integral Comunitario Integral Ayuujk Polivalente (BICAP): una experiencia mixe de educación compleja. Revista Iberoamericana para la Investigación y el Desarrollo Educativo, publicación 10. Recuperado de http://ride.org.mx/1-11/index.php/RIDESECUNDARIO/article/view/336/328

Motola, S. (2009, julio). Protesta pedagógica: educación alternativa creada por y para la gente de los pueblos originarios de Oaxaca que preservan la heterogeneidad de la cultura mexicana. Educación Comunal, núm. 2, pp.17-37.

Pardo, E. y Roddo, R. (2008). Desarrollo del saber local en el trabajo educativo. En Diálogo de saberes y escuela rural andina (pp. 107-126). Lima: Proyecto Andino de Tecnologías Campesinas.

Pardo, T. (2009). El contexto de la experiencia educativa del Instituto Comunitario Mixe Kong Oy. Cuadernos del Sur, núm. 27, pp. 45-52.

Pastrana, D. (2010). Universidad Ayuuk, educación desde las culturas indígenas. Recuperado de http://www.magis.iteso.mx/content/universidad-ayuuk

Pérez, F. (2008). El Bachillerato Integral Comunitario, un modelo educativo de nivel medio superior de los Pueblos Originarios en Oaxaca, México: un análisis curricular. Tesis de maestría en Comunicación Educativa, CECTE-ILCE, México.

Rengifo, G. (2005). Explorando caminos para la incorporación del saber local en la escuela. En PRATEC. Saber local en la comunidad y en la escuela (pp. 13-18). Lima: Proyecto Andino de Tecnologías Campesinas.

Reyes, S. y Vásquez, B. (2008). Formar en la diversidad: el caso de la Escuela Normal 
Bilingüe e Intercultural de Oaxaca (ENBIO). Trace, núm. 53, pp. 83-99.

Ricco, S. y Rebolledo, N. (2010). Educación y comunalidad: prácticas autonómicas en la Mazateca Alta. México: UPN.

Rioja, E. (2013). Bachilleratos comunitarios, una forma alternativa de hacer educación. Tlathoa, núm. 10, pp. 20-31. Recuperado de file:///C:/Users/admin/Downloads/tlahtoa10.pdf

Rodríguez, J. R. (2014). Los egresados del Instituto Superior Intercultural ISIA-Ayuuk y sus perspectivas laborales en la zona del Bajo Mixe. Oaxaca Población Siglo XXI, vol. 14, núm. 37, pp. 14-25. Recuperado de http://www.digepo.oaxaca.gob.mx/ recursos/revistas/revista37.pdf

Ruiz, A. y Quiroz, E. (2014). Educación comunitaria: una propuesta alternativa para los pueblos indígenas de Oaxaca-México. Polis, núm. 38, pp. 83-99. Recuperado de https://polis.revues.org/10107

Sanabria, L. (2015). Sistematización de la experiencia educativa del Colectivo Utopía (Oaxaca, México) (video). Recuperado de http://www.cepalforja.org/sistem/ bvirtual/?p=1068

SEP (2017). Ruta para la implementación del modelo educativo. México.

Slutsky-Moore, R. (2011). ¿Aprendemos juntos? Guiando la educación comunitaria en Oaxaca. Educación Comunal, núm. 4-5, pp. 117-144.

Vargas, M. (2005). Nunca más un México sin nosotros. Expresiones etnopolíticas oaxaqueñas. México: INAH.

Vargas, X. (2014). Wejën kajën: sociedad, cultura y educación del pueblo originario Ayuujk del estado de Oaxaca, México. Una aportación a la formación maduración armónica del ser humano pueblo frente a los retos del siglo XXI. Oaxaca Población Siglo XXI, vol. 14, núm. 37, pp. 26-31. Recuperado de http://www.digepo.oaxaca. gob.mx/recursos/revistas/revista37.pdf

Vargas, X., Vargas, P., Vásquez, R. y Pérez, L. (comps.) (2008). Wejën-Kajën. Las dimensiones del pensamiento y generación del conocimiento comunal. Tlahuitoltepec: H. Ayuntamiento Único Constitucional de Tlahuitoltepec, Mixe, Oaxaca.

Wagner, J. (2008). La Escuela Secundaria Bilingüe Comunitaria en San Pedro Yaneri, Ixtlán. Educación Comunal, núm. 1, pp. 71-81.

Weiss, E. (2017). Estudio exploratorio del Modelo de Telebachillerato Comunitario y su operación en los estados. México: INEE. Recuperado de http://publicaciones.inee. edu.mx/buscadorPub/P1/C/155/P1C155.pdf 